Check for updates FAST TRACK
OPEN ACCESS

\section{Early initiation of prophylactic anticoagulation for prevention of coronavirus disease 2019 mortality in patients admitted to hospital in the United States: cohort study}

\author{
Christopher T Rentsch, ${ }^{1,2}$ Joshua A Beckman, ${ }^{3}$ Laurie Tomlinson, ${ }^{1}$ Walid F Gellad,,${ }^{4,5,6}$ \\ Charles Alcorn, ${ }^{7}$ Farah Kidwai-Khan, ${ }^{2,8}$ Melissa Skanderson, ${ }^{2}$ Evan Brittain, ${ }^{9}$ \\ Joseph T King Jr, ${ }^{2,10}$ Yuk-Lam Ho, ${ }^{11}$ Svetlana Eden, ${ }^{12}$ Suman Kundu, ${ }^{13}$ Michael F Lann, ${ }^{7}$ \\ Robert A Greevy Jr, ${ }^{14}$ P Michael Ho, ${ }^{15}$ Paul A Heidenreich, ${ }^{16,17}$ Daniel A Jacobson, ${ }^{18}$ \\ Ian J Douglas, ${ }^{1}$ Janet P Tate, ${ }^{2,8}$ Stephen J W Evans, ${ }^{1}$ David Atkins, ${ }^{19}$ Amy C Justice, ${ }^{2,8,20}$ \\ Matthew S Freiberg ${ }^{13,21}$
}

For numbered affiliations see end of the article. christopher.rentsch@lshtm.ac.uk (or @darthctr on Twitter: ORCID 0000-0002-1408-7907) Additional material is published online only. To view please visit the journal online.

Cite this as: $B M / 2021 ; 372: n 311$ http://dx.doi.org/10.1136/bmi.n311

Accepted: 1 February 2021
Correspondence to: CT Rentsch

\section{ABSTRACT \\ OBJECTIVE}

To evaluate whether early initiation of prophylactic anticoagulation compared with no anticoagulation was associated with decreased risk of death among patients admitted to hospital with coronavirus disease 2019 (covid-19) in the United States.

DESIGN

Observational cohort study.

SETTING

Nationwide cohort of patients receiving care in the Department of Veterans Affairs, a large integrated national healthcare system.

\section{PARTICIPANTS}

All 4297 patients admitted to hospital from 1 March to 31 July 2020 with laboratory confirmed severe acute respiratory syndrome coronavirus 2 (SARS-CoV-2) infection and without a history of anticoagulation.

MAIN OUTCOME MEASURES

The main outcome was 30 day mortality. Secondary outcomes were inpatient mortality, initiating therapeutic anticoagulation (a proxy for clinical

\section{WHAT IS ALREADY KNOWN ON THIS TOPIC}

Deaths among patients with coronavirus disease 2019 (covid-19) are partially attributed to venous thromboembolism and arterial thromboses

Anticoagulants prevent thrombosis formation, possess antiviral and potentially anti-inflammatory properties, and might be particularly effective in patients with covid-19

Evaluations of the efficacy of prophylactic anticoagulation in patients with covid-19 in randomized clinical trials are underway but not yet reported; previous observational studies have been limited in sample size or used relatively small healthcare systems

\section{WHAT THIS STUDY ADDS}

This study found that initiation of prophylactic anticoagulation compared with no anticoagulation within 24 hours of hospital admission was associated with a relative risk reduction of 30 day mortality as high as $34 \%$ and an absolute risk reduction of $4.4 \%$ among patients admitted to hospital with covid-19

In a post hoc safety analysis, receipt of prophylactic anticoagulation was not associated with increased risk of bleeding that required transfusion

These findings provide strong real world evidence to support guidelines recommending the use of prophylactic anticoagulation as initial treatment for patients with covid-19 on hospital admission deterioration, including thromboembolic events), and bleeding that required transfusion.

\section{RESULTS}

Of 4297 patients admitted to hospital with covid-19, 3627 (84.4\%) received prophylactic anticoagulation within 24 hours of admission. More than $99 \%$ $(n=3600)$ of treated patients received subcutaneous heparin or enoxaparin. 622 deaths occurred within 30 days of hospital admission, 513 among those who received prophylactic anticoagulation. Most deaths (510/622, 82\%) occurred during hospital stay. Using inverse probability of treatment weighted analyses, the cumulative incidence of mortality at 30 days was $14.3 \%$ (95\% confidence interval $13.1 \%$ to $15.5 \%)$ among those who received prophylactic anticoagulation and $18.7 \%$ (15.1\% to $22.9 \%$ ) among those who did not. Compared with patients who did not receive prophylactic anticoagulation, those who did had a $27 \%$ decreased risk for 30 day mortality (hazard ratio $0.73,95 \%$ confidence interval 0.66 to 0.81). Similar associations were found for inpatient mortality and initiation of therapeutic anticoagulation. Receipt of prophylactic anticoagulation was not associated with increased risk of bleeding that required transfusion (hazard ratio $0.87,0.71$ to 1.05 ). Quantitative bias analysis showed that results were robust to unmeasured confounding (e-value lower $95 \%$ confidence interval 1.77 for 30 day mortality). Results persisted in several sensitivity analyses.

\section{CONCLUSIONS}

Early initiation of prophylactic anticoagulation compared with no anticoagulation among patients admitted to hospital with covid-19 was associated with a decreased risk of 30 day mortality and no increased risk of serious bleeding events. These findings provide strong real world evidence to support guidelines recommending the use of prophylactic anticoagulation as initial treatment for patients with covid-19 on hospital admission.

\section{Introduction}

Severe acute respiratory syndrome coronavirus 2 (SARSCoV-2), the virus that causes coronavirus disease 2019 (covid-19), continues to spread worldwide. Deaths among people with covid-19 have been partially attributed to venous thromboembolism and arterial thromboses. ${ }^{12}$ In intensive care settings, prevalence 
of venous thromboembolism among patients with covid-19 has been reported at about $30 \% .^{3}$ In response, several expert organizations, including the American Society of Hematology, ${ }^{4}$ International Society on Thrombosis and Haemostasis, ${ }^{5}$ CHEST Guideline and Expert Panel, ${ }^{6}$ and others ${ }^{17}$ have recommended prophylactic anticoagulation for patients admitted with covid-19, who do not have a contraindication to this treatment, to reduce the risk of thromboembolism.

Heparin based anticoagulants are commonly used in hospital settings. Given evidence that shows these drugs might also possess anti-inflammatory properties, ${ }^{8-10}$ heparin-based treatments might be particularly effective in patients with covid-19. ${ }^{11}$ Randomized clinical trials evaluating the efficacy of prophylactic anticoagulation in patients with covid-19 are underway. ${ }^{12}$ Previous observational cohort studies have found evidence that use of anticoagulation in patients with covid-19 was associated with decreased risk of mortality ${ }^{13}{ }^{14}$; however, these studies were limited in sample size or used relatively small healthcare systems. In this study we estimated the effect of early initiation of prophylactic anticoagulation compared with no anticoagulation on risk of 30 day mortality among patients admitted to hospital with covid-19 in the largest integrated healthcare system in the United States.

\section{Methods}

This study is reported according to the strengthening the reporting of observational studies in epidemiology (STROBE) and reporting of studies conducted using observational routinely collected health data (RECORD) guidelines (see supplementary appendix).

\section{Study design and population}

We conducted an observational cohort study using electronic health record data from the US Department of Veterans Affairs, which comprises more than 1200 points of care nationwide, including hospitals, medical centers, and community outpatient clinics. All care is recorded in a central data repository, with daily uploads into the Veterans Affairs Corporate Data Warehouse. Available data include demographics, outpatient and inpatient encounters, diagnoses, procedures, smoking and alcohol health behaviors, pharmacy dispensing records, vital signs, laboratory measures, and death information.

We included all patients admitted to hospital between 1 March and 31 July 2020 who had a laboratory confirmed positive SARS-CoV-2 test result on or within 14 days before hospital admission. We excluded patients who had no history of care (defined as at least one outpatient or inpatient encounter in the two years before 1 March 2020), received anticoagulation treatment in the 30 days before hospital admission (to mitigate the effect of prevalent use of anticoagulation), received a red blood cell transfusion within 24 hours of admission (as active bleeding or severe anemia could have been a contraindication for anticoagulation), or experienced any outcome within 24 hours of admission and therefore did not have equal chance to be classified as having received anticoagulation in this study.

\section{Types and doses of anticoagulants}

We extracted inpatient pharmacy records for warfarin, intravenous heparin, low molecular weight heparin (enoxaparin, fondaparinux, dalteparin), and direct oral anticoagulants (apixaban, rivaroxaban, dabigatran). Doses and routes considered prophylactic and therapeutic anticoagulation are listed in supplementary box 1 in the appendix.

\section{Anticoagulation, outcomes, and follow-up}

We compared prophylactic anticoagulation in the first 24 hours of hospital admission with no receipt of anticoagulation in the same time frame. The primary outcome was mortality within 30 days of hospital admission, which included in-hospital deaths (those during hospital admission) and those that occurred after discharge. Secondary outcomes were inpatient mortality and initiation of therapeutic anticoagulation. Algorithms to identify thromboembolic events during hospital admission of patients with covid-19 have yet to be validated; thus, we considered initiation of therapeutic levels of anticoagulation after the first 24 hours of admission to be a proxy for clinical deterioration, including thromboembolic events. For all outcomes, we followed patients from date of hospital admission until the earliest date of the outcome, a maximum of 30 days, or 30 August 2020.

Some Veterans Affairs hospitals report observation periods and admissions separately, even when patients have not moved beds or changed providers. We combined these periods and considered a full hospital admission to begin at first presentation in a Veterans Affairs hospital and end when there was no subsequent hospital stay that began within 24 hours. Figure 1 shows the study design.

\section{Covariates}

Potential confounders in the relation between prophylactic anticoagulation and covid-19 mortality or thromboembolic events were identified by review of the existing literature and discussions with clinicians. We extracted information on age, race, ethnicity, sex, urban or rural residence, US census region, clinical comorbidities, Charlson comorbidity index, and substance use. Presence of clinical comorbidities was determined by one inpatient or two outpatient diagnoses using ICD-9 or ICD-10 (international classification of diseases, ninth or 10th revision) codes in the two years before hospital admission, except for cancer, which was considered present if diagnosed ever before hospital admission. Level of alcohol consumption was calculated using the most recent alcohol use disorder identification testconsumption (AUDIT-C) ${ }^{15}$ measure within two years before admission. Smoking status was determined by the most frequent response in the five years before hospital admission. ${ }^{16}$ 


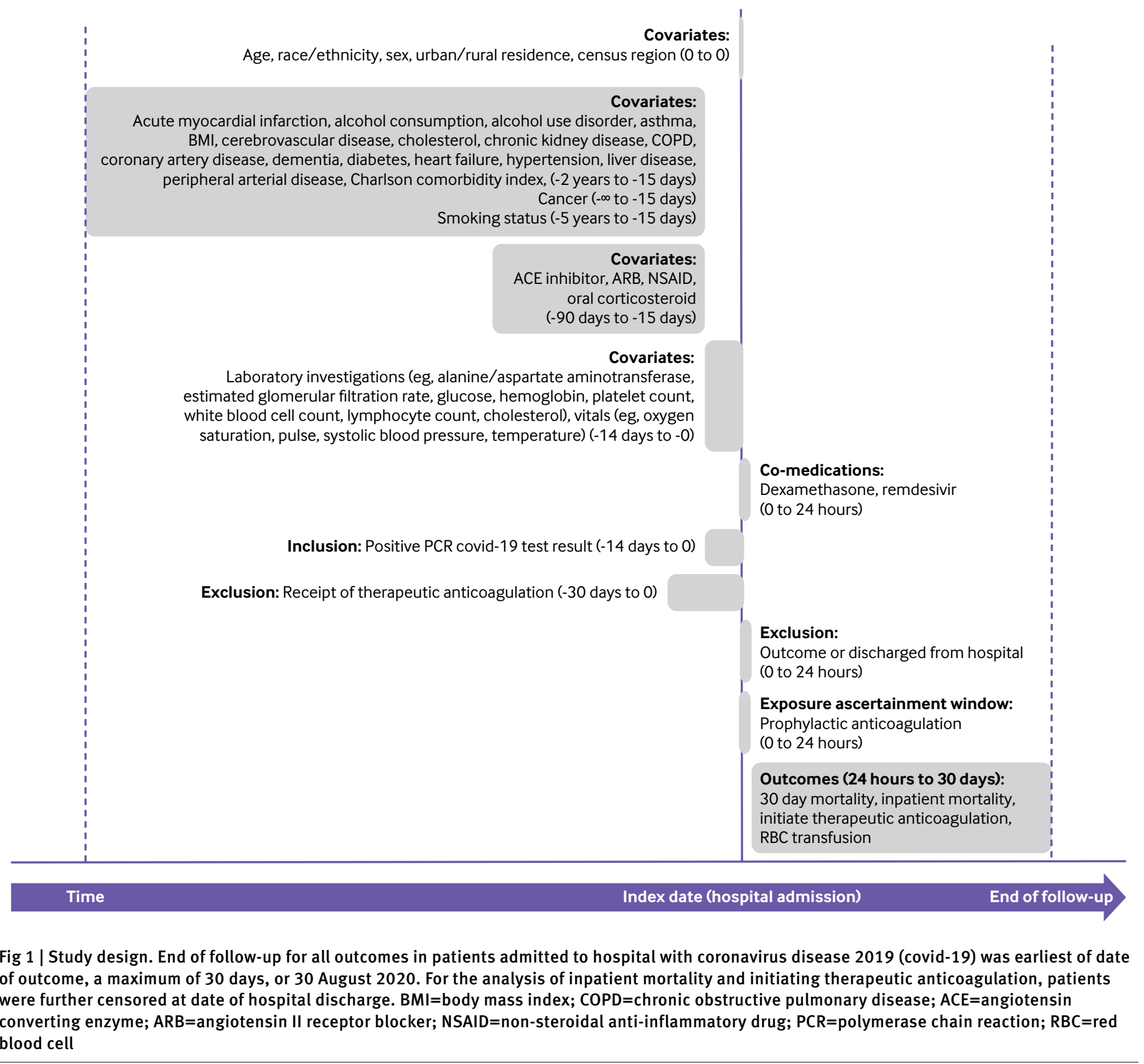

We ascertained drug history pertinent to the 15 to 90 days before hospital admission. To account for acute health status at hospital admission, we captured vital signs and laboratory measures. Body mass index was calculated from height and weight measurements closest to hospital admission within five and two years, respectively, before admission. All other vital signs and all laboratory measures utilized the value closest to hospital admission within 14 days before admission. Figure 1 provides further details on covariate ascertainment windows. Supplementary box 2 in the appendix provides a list of potentially relevant covariates that could not be included owing to unavailability or high levels of missingness, including inflammatory markers $\mathrm{D}$ dimer and $\mathrm{C}$ reactive protein.
To account for potential effects of co-medications with other covid-19 treatments, we ascertained whether patients had received oral or intravenous dexamethasone ${ }^{17}$ at any dose, or intravenous remdesivir $^{18}$ at any dose within the first 24 hours of hospital admission as well as treatments received after the first 24 hours.

Covariates with the largest proportion of missing data included alanine aminotransferase (13.5\%), aspartate aminotransferase (15.2\%), lymphocyte count (15.0\%), and total cholesterol (14.1\%): all other covariates had less than $10 \%$ of data missing.

\section{Propensity score model}

We used inverse probability of treatment weighting to estimate the average treatment effect. This contrast 
was chosen because we excluded patients with contraindications to prophylactic anticoagulation, and thus assume all included patients were eligible for anticoagulation. We first modeled the probability of receiving anticoagulation as a function of all measured covariates (apart from in-hospital treatments received after the first 24 hours so as to not use future information at baseline). ${ }^{19}$ Propensity scores (ie, the predicted probability of exposure) were estimated using a multivariable logistic regression model. Of 45 variables in the model, only four had missingness between $10 \%$ and $15 \%$; all others had $10 \%$ or less, and most (30 of 45 variables) were complete. We included a missing category for covariates with missing data given the variables with most missingness do not drive the decision to administer prophylactic anticoagulation, but rather are markers for general health severity. Under the additional assumption that associations between fully observed covariates and receipt of prophylactic anticoagulation did not differ across missingness patterns, this approach produces unbiased estimates. ${ }^{20} 21$ Each patient was weighted by the inverse probability of receiving the exposure of interest, with the goal of balancing observable characteristics, including missingness patterns, ${ }^{22}$ between treatment groups. After inverse probability of treatment weighting, the distribution of propensity scores between the treatment groups overlapped nearly perfectly (see supplementary eFigure 1). We calculated absolute standardized mean differences between treatment groups and considered 0.2 or less as balanced, ${ }^{23}$ although most were 0.1 or less (see supplementary eFigure 2). Thus, the weighting produced treatment groups that were considered well balanced.

\section{Statistical analysis}

Covariates were summarized using descriptive statistics, stratified by treatment group. We calculated the inverse probability of treatment weighted sample sizes by multiplying weights by constant factor $\mathrm{k}$, where $\mathrm{k}$ was the ratio of observed sample size to number in the pseudopopulation after weighting; in this study, $k=4297 / 8576$. This scaling was performed to force the sample size in the pseudopopulation after weighting to equal the sample size in the observed population. We generated inverse probability of treatment weighted Kaplan-Meier plots. Cox regression models with days since hospital admission as the timescale were used to estimate inverse probability of treatment weighted hazard ratios and 95\% confidence intervals for the effect of early initiation of prophylactic doses of anticoagulation compared with no anticoagulation on 30 day mortality, inpatient mortality, and initiating therapeutic anticoagulation. Both secondary outcomes occur during hospital admission, wherein discharge from hospital was considered a competing event. If patients were censored at discharge, absolute risks derived from Kaplan-Meier analyses would be overestimated. ${ }^{2425}$ We therefore displayed cumulative incidence rates by treatment group, treating discharge as a competing event (ie, no censoring at date of discharge). As our question was causal in nature, we nonetheless chose to display cause specific rather than subdistribution hazard ratios. ${ }^{26}$ These cause specific hazard ratios were interpreted as the effect of prophylactic anticoagulation compared with no anticoagulation on each of the outcomes irrespective of the effect on discharge. Proportional hazards were checked by examining the complementary log-log (or the log of negative log) of estimated survivor functions for groups that received or did not receive prophylactic anticoagulation versus the log of survival time. No evidence was found of proportional hazards violations. We used Microsoft SQL Server Management Studio v17.4 for data management and SAS version 9.4 (SAS Institute, Cary, NC) and Stata 16 MP for statistical analyses.

\section{Sensitivity analyses}

For each outcome we used quantitative bias analysis to calculate an e-value, which demonstrates the strength of association between an unmeasured confounder and exposure or outcome, conditional on measured covariates that would be necessary to fully explain observed effects. ${ }^{27}$ To assess for undue effects from outliers with very high or very low estimated propensity of treatment, we capped propensity score distributions at the 1st and 99th centiles and again at the 5th and 95th centiles. To account for potentially biased estimation of standard errors or influence from very high or very low weights, we performed sensitivity analyses using combinations of robust standard error estimation ${ }^{28}$ and stabilized weighting. ${ }^{29}$ We re-ran the primary analyses extending the exposure ascertainment window from 24 to 48 hours. Given the low frequency for use of direct oral anticoagulants in the cohort, we re-ran analyses excluding these drugs from the treated group.

\section{Post hoc analyses}

In post hoc analyses, we assessed the effect of prophylactic anticoagulation separately by the two most prescribed drugs in the cohort: subcutaneous heparin and enoxaparin. We stratified the primary model by whether patients were admitted to the intensive care unit (ICU) within the first 24 hours of hospital admission. To investigate safety associated with prophylactic anticoagulation, we fitted an additional model with bleeding that required red blood cell transfusion as the outcome.

\section{Patient and public involvement}

Patients were not consulted during the initial design of the study. However, in the course of peer review, we received thoughtful comments from a patient and carer and revised our manuscript accordingly. In addition, we engaged with the Veterans Affairs central office and chief medical officers during the entire course of this research. 


\section{Results}

Overall, 4297 patients admitted to hospital with covid-19 between 1 March and 31 July 2020 were included in this analysis (fig 2). Median age in the cohort was 68 years (interquartile range 58-75 years); most patients were of non-Hispanic black $(n=1940$, 45.1\%), non-Hispanic white $(\mathrm{n}=1603,37.3 \%)$, and Hispanic $(n=506,11.8 \%)$ race or ethnicity. Most of the patients were men $(n=4015,93.4 \%)$, located in the south $(n=2017,46.9 \%)$, and resident in an urban area $(n=3768,87.7 \%)$ (table 1$)$. More patients were admitted to hospital in July ( $\mathrm{n}=1401,32.6 \%)$ than any other month.

In this cohort, $3627(84.4 \%)$ patients received prophylactic anticoagulation within 24 hours of hospital admission. Among those who received prophylactic anticoagulation, the most common drugs were heparin based: either subcutaneous heparin $(n=1094,30.2 \%)$ or enoxaparin $(n=2506,69.1 \%)$.

At hospital presentation, the group of patients who received prophylactic anticoagulation, compared with the group of patients who did not, had a higher proportion with an oxygen saturation level less than $93 \%$ (table $1 ; 16.0 \% \vee 10.7 \%$ ), heart rate at 90 beats/ min or higher $(39.3 \% \vee 34.6 \%)$, and temperature of $38^{\circ} \mathrm{C}$ or higher $(17.5 \% v 10.4 \%)$. In contrast, the burden of prevalent comorbid disease (Charlson comorbidity index score 25 ) was lower among those who received prophylactic anticoagulation compared with those who did not $(21.1 \% v 25.1 \%)$. Treatment for covid-19 using other drugs within the first 24 hours of hospital admission were more common among those who received prophylactic anticoagulation compared with those who did not (16.2\% $v 11.0 \%$ for dexamethasone; $12.0 \% \vee 5.2 \%$ for remdesivir). After inverse probability of treatment weighting, however, differences were minimized between the two treatment groups (all standardized mean differences $\leq 0.2$, with most $\leq 0.1$; table 1 and table 2 and supplementary eFigure 2).

\section{Absolute and relative risks}

Overall, 622 deaths $(622 / 4297,14.5 \%)$ occurred within 30 days of hospital admission, 513 among those who received prophylactic anticoagulation (table 3). Most deaths (510/622, 82\%) occurred during hospital admission. In inverse probability of treatment weighted analyses, the cumulative incidence of mortality at 30 days was $14.3 \%$ (95\% confidence interval $13.1 \%$ to $15.5 \%$ ) for patients receiving prophylactic anticoagulation and $18.7 \%$ (15.1\% to 22.9\%) for those receiving no anticoagulation (table 3). Receiving prophylactic anticoagulation was associated with a $27 \%$ decreased risk of death over the first 30 days (hazard ratio $0.73,95 \%$ confidence interval 0.66 to 0.81 ; table 3 and fig 3) compared with receiving no anticoagulation. Similar associations were found for inpatient mortality $(0.69,0.61$ to 0.77$)$ and initiating therapeutic anticoagulation (0.81, 0.73 to 0.90$)$.

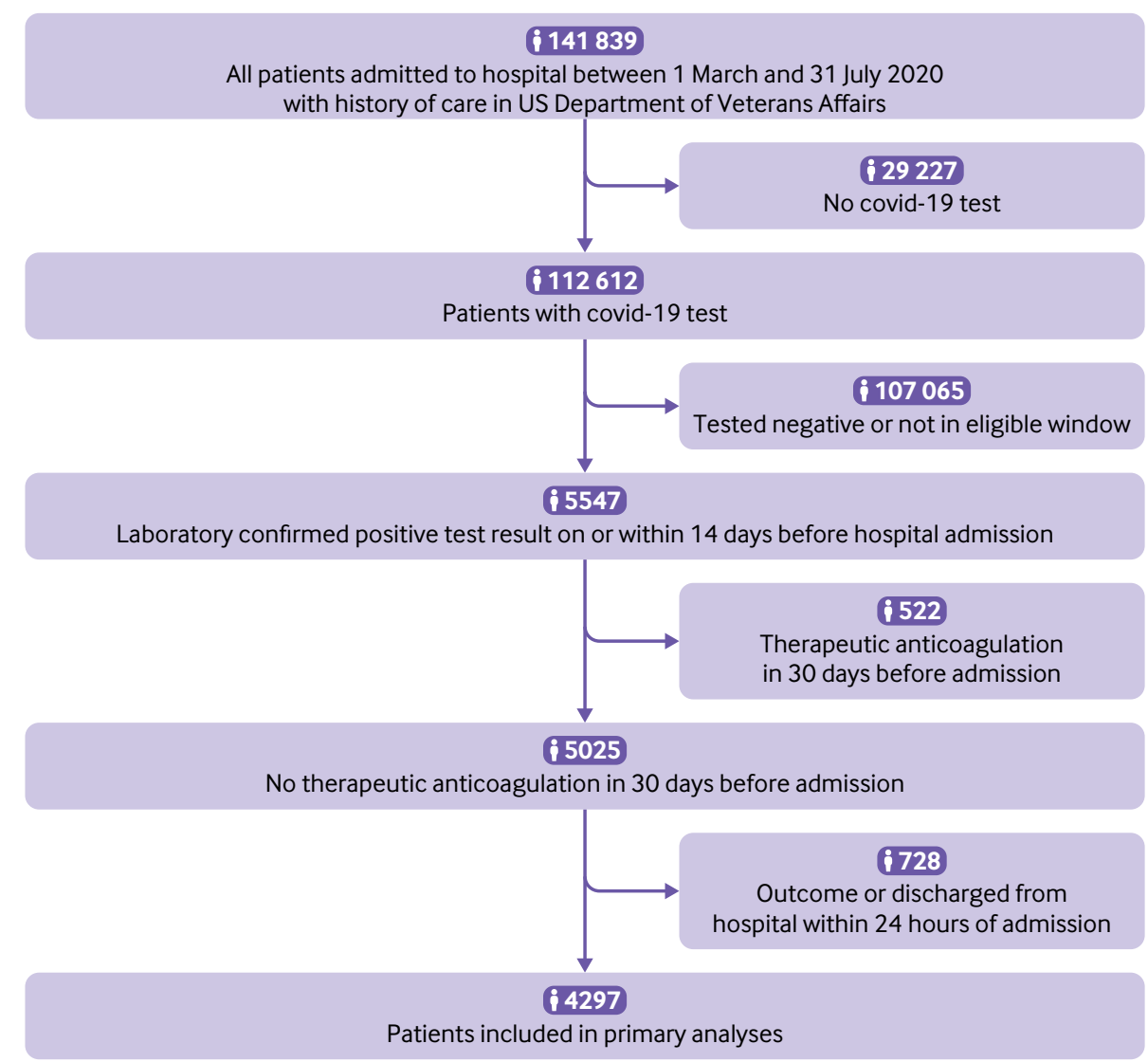

Fig 2 | Flow chart of patients admitted to hospital with coronavirus disease 2019 (covid-19) 


\begin{tabular}{|c|c|c|c|c|c|c|}
\hline \multirow[b]{2}{*}{ Characteristics } & \multicolumn{3}{|c|}{ Unweighted } & \multicolumn{3}{|c|}{ IPT weighted* } \\
\hline & $\begin{array}{l}\text { No anticoagulation } \\
(\mathrm{n}=670)\end{array}$ & $\begin{array}{l}\text { Prophylactic } \\
\text { anticoagulation }(n=3627)\end{array}$ & SMD & $\begin{array}{l}\text { No anticoagulation } \\
(n=2141)\end{array}$ & $\begin{array}{l}\text { Prophylactic } \\
\text { anticoagulation }(n=2156)\end{array}$ & SMD \\
\hline \multicolumn{7}{|l|}{ Personal characteristics } \\
\hline Median (interquartile range) age (years) & $69.0(58.0-76.5)$ & $68.1(58.2-74.8)$ & 0.04 & $69.4(59.7-76.5)$ & $68.3(58.2-75.0)$ & 0.04 \\
\hline \multicolumn{7}{|l|}{ Age groups (years): } \\
\hline $20-49$ & $89(13.3)$ & $446(12.3)$ & 0.03 & $239(11.2)$ & $265(12.3)$ & 0.04 \\
\hline $50-59$ & $105(15.7)$ & $619(17.1)$ & 0.04 & $313(14.6)$ & $363(16.8)$ & 0.06 \\
\hline $60-69$ & $161(24.0)$ & $951(26.2)$ & 0.05 & $554(25.9)$ & $558(25.9)$ & 0.00 \\
\hline $70-79$ & $188(28.1)$ & $1056(29.1)$ & 0.02 & $652(30.5)$ & $626(29.0)$ & 0.03 \\
\hline$\geq 80$ & $127(19.0)$ & $555(15.3)$ & 0.10 & $383(17.9)$ & $343(15.9)$ & 0.05 \\
\hline \multicolumn{7}{|l|}{ Race or ethnicity: } \\
\hline White & $256(38.2)$ & $1347(37.1)$ & 0.02 & $832(38.9)$ & $806(37.4)$ & 0.03 \\
\hline Black & $291(43.4)$ & $1649(45.5)$ & 0.04 & $940(43.9)$ & $974(45.2)$ & 0.03 \\
\hline Hispanic & $74(11.0)$ & $432(11.9)$ & 0.03 & $238(11.1)$ & $252(11.7)$ & 0.02 \\
\hline Other & $22(3.3)$ & $102(2.8)$ & 0.03 & $76(3.6)$ & $63(2.9)$ & 0.04 \\
\hline Unknown & $27(4.0)$ & $97(2.7)$ & 0.08 & $55(2.6)$ & $61(2.8)$ & 0.02 \\
\hline Men & $620(92.5)$ & 3395 (93.6) & 0.04 & 2019 (94.3) & $2014(93.4)$ & 0.04 \\
\hline Urban residence & $587(87.6)$ & $3181(87.7)$ & 0.00 & $1915(89.5)$ & $1893(87.8)$ & 0.05 \\
\hline \multicolumn{7}{|l|}{ Census region: } \\
\hline Midwest & $79(11.8)$ & $724(20.0)$ & 0.22 & $343(16.0)$ & $400(18.6)$ & 0.07 \\
\hline Northeast & $139(20.7)$ & $622(17.1)$ & 0.09 & $432(20.2)$ & $383(17.8)$ & 0.06 \\
\hline South & $314(46.9)$ & $1703(47.0)$ & 0.00 & $998(46.6)$ & $1012(46.9)$ & 0.01 \\
\hline West & $138(20.6)$ & $578(15.9)$ & 0.12 & $367(17.2)$ & $361(16.7)$ & 0.01 \\
\hline \multicolumn{7}{|l|}{ Month of admission: } \\
\hline March & $116(17.3)$ & $518(14.3)$ & 0.08 & $323(15.1)$ & $316(14.7)$ & 0.01 \\
\hline April & $169(25.2)$ & 868 (23.9) & 0.03 & $491(22.9)$ & $522(24.2)$ & 0.03 \\
\hline May & $70(10.4)$ & $429(11.8)$ & 0.04 & $277(12.9)$ & $250(11.6)$ & 0.04 \\
\hline June & $110(16.4)$ & $616(17.0)$ & 0.02 & $361(16.9)$ & $364(16.9)$ & 0.00 \\
\hline July & $205(30.6)$ & $1196(33.0)$ & 0.05 & $689(32.2)$ & $704(32.6)$ & 0.01 \\
\hline \multicolumn{7}{|l|}{ Clinical conditions } \\
\hline Acute myocardial infarction & $11(1.6)$ & $66(1.8)$ & 0.01 & $45(2.1)$ & $39(1.8)$ & 0.02 \\
\hline Asthma & $33(4.9)$ & $176(4.9)$ & 0.00 & $117(5.5)$ & $105(4.9)$ & 0.03 \\
\hline Cancer, any & $97(14.5)$ & $494(13.6)$ & 0.02 & $318(14.9)$ & $298(13.8)$ & 0.03 \\
\hline Cerebrovascular disease & $85(12.7)$ & $369(10.2)$ & 0.08 & $223(10.4)$ & $230(10.7)$ & 0.01 \\
\hline Chronic kidney disease & $136(20.3)$ & $694(19.1)$ & 0.03 & $436(20.4)$ & $421(19.5)$ & 0.02 \\
\hline COPD & $105(15.7)$ & $544(15.0)$ & 0.02 & $359(16.8)$ & $328(15.2)$ & 0.04 \\
\hline Coronary artery disease & $25(3.7)$ & $90(2.5)$ & 0.07 & $65(3.0)$ & $59(2.7)$ & 0.02 \\
\hline Dementia & $104(15.5)$ & $378(10.4)$ & 0.15 & $261(12.2)$ & $244(11.3)$ & 0.03 \\
\hline Diabetes & $269(40.1)$ & $1573(43.4)$ & 0.07 & $859(40.1)$ & $924(42.9)$ & 0.06 \\
\hline Heart failure & $77(11.5)$ & $375(10.3)$ & 0.04 & $265(12.4)$ & $232(10.8)$ & 0.05 \\
\hline Hypertension & $446(66.6)$ & $2470(68.1)$ & 0.03 & $1380(64.5)$ & $1462(67.8)$ & 0.07 \\
\hline Liver disease & $71(10.6)$ & $322(8.9)$ & 0.06 & $209(9.8)$ & $199(9.2)$ & 0.02 \\
\hline Peripheral arterial disease & $70(10.4)$ & $387(10.7)$ & 0.01 & $236(11.0)$ & $229(10.6)$ & 0.01 \\
\hline \multicolumn{7}{|l|}{ Charlson comorbidity index score: } \\
\hline 0 & $130(19.4)$ & $765(21.1)$ & 0.04 & $404(18.9)$ & $450(20.9)$ & 0.05 \\
\hline 1 & $110(16.4)$ & $723(19.9)$ & 0.09 & $388(18.1)$ & $423(19.6)$ & 0.04 \\
\hline 2 & $119(17.8)$ & $657(18.1)$ & 0.01 & $403(18.8)$ & $384(17.8)$ & 0.03 \\
\hline 3 & $74(11.0)$ & $394(10.9)$ & 0.01 & $252(11.8)$ & $235(10.9)$ & 0.03 \\
\hline 4 & $69(10.3)$ & $324(8.9)$ & 0.05 & $217(10.2)$ & $194(9.0)$ & 0.04 \\
\hline$\geq 5$ & $168(25.1)$ & $764(21.1)$ & 0.10 & $476(22.2)$ & $470(21.8)$ & 0.01 \\
\hline \multicolumn{7}{|l|}{ Drug history } \\
\hline ACE inhibitor & $119(17.8)$ & $807(22.2)$ & 0.11 & $422(19.7)$ & $463(21.5)$ & 0.04 \\
\hline ARB & $78(11.6)$ & $481(13.3)$ & 0.05 & $261(12.2)$ & $283(13.1)$ & 0.03 \\
\hline NSAID & $144(21.5)$ & $731(20.2)$ & 0.03 & $408(19.1)$ & $438(20.3)$ & 0.03 \\
\hline Oral corticosteroid & $156(23.3)$ & $875(24.1)$ & 0.02 & $514(24.0)$ & $516(24.0)$ & 0.00 \\
\hline \multicolumn{7}{|l|}{ In-hospital treatments } \\
\hline \multicolumn{7}{|l|}{ Dexamethasone: } \\
\hline$<24$ hours & $74(11.0)$ & $588(16.2)$ & 0.15 & $309(14.4)$ & $332(15.4)$ & 0.03 \\
\hline$>24$ hours & $115(17.2)$ & $892(24.6)$ & 0.18 & $463(21.6)$ & $508(23.6)$ & 0.05 \\
\hline \multicolumn{7}{|l|}{ Remdesivir: } \\
\hline$<24$ hours & $35(5.2)$ & $437(12.0)$ & 0.24 & $204(9.5)$ & $236(10.9)$ & 0.05 \\
\hline$>24$ hours & 89 (13.3) & $791(21.8)$ & 0.23 & 341 (15.9) & $447(20.7)$ & 0.12 \\
\hline
\end{tabular}




\begin{tabular}{|c|c|c|c|c|c|c|}
\hline \multirow[b]{2}{*}{ Characteristics } & \multicolumn{3}{|c|}{ Unweighted } & \multicolumn{3}{|c|}{ IPT weighted* } \\
\hline & $\begin{array}{l}\text { No anticoagulation } \\
(n=670)\end{array}$ & $\begin{array}{l}\text { Prophylactic } \\
\text { anticoagulation }(n=3627)\end{array}$ & SMD & $\begin{array}{l}\text { No anticoagulation } \\
(n=2141)\end{array}$ & $\begin{array}{l}\text { Prophylactic } \\
\text { anticoagulation }(n=2156)\end{array}$ & SMD \\
\hline \multicolumn{7}{|l|}{ Substance use } \\
\hline \multicolumn{7}{|c|}{ Alcohol consumption status: } \\
\hline Abstinent & $51(7.6)$ & $300(8.3)$ & 0.02 & $178(8.3)$ & $177(8.2)$ & 0.00 \\
\hline Low risk & $360(53.7)$ & $1831(50.5)$ & 0.07 & $1059(49.4)$ & $1098(50.9)$ & 0.03 \\
\hline At risk & $148(22.1)$ & $965(26.6)$ & 0.11 & $574(26.8)$ & $557(25.8)$ & 0.02 \\
\hline Hazardous & $28(4.2)$ & $161(4.4)$ & 0.01 & $79(3.7)$ & $94(4.4)$ & 0.03 \\
\hline Alcohol use disorder & $3(0.4)$ & $19(0.5)$ & 0.01 & $11(0.5)$ & $11(0.5)$ & 0.00 \\
\hline Missing & 80 (11.9) & $351(9.7)$ & 0.07 & $241(11.3)$ & $219(10.1)$ & 0.04 \\
\hline \multicolumn{7}{|l|}{ Smoking status: } \\
\hline Never & $17(2.5)$ & $63(1.7)$ & 0.06 & $42(2.0)$ & 41 (1.9) & 0.00 \\
\hline Former & $258(38.5)$ & $1431(39.5)$ & 0.02 & $753(35.1)$ & $842(39.1)$ & 0.08 \\
\hline Current & $225(33.6)$ & $1355(37.4)$ & 0.08 & $905(42.3)$ & $793(36.8)$ & 0.11 \\
\hline Missing & $170(25.4)$ & $778(21.5)$ & 0.09 & $442(20.6)$ & $479(22.2)$ & 0.04 \\
\hline \multicolumn{7}{|l|}{ Vital signs } \\
\hline \multicolumn{7}{|l|}{ Body mass index: } \\
\hline$<26$ & $206(30.7)$ & $938(25.9)$ & 0.11 & $630(29.4)$ & $576(26.7)$ & 0.06 \\
\hline $26-32$ & $258(38.5)$ & $1436(39.6)$ & 0.02 & $940(43.9)$ & $854(39.6)$ & 0.09 \\
\hline$\geq 33$ & $169(25.2)$ & $1113(30.7)$ & 0.12 & $494(23.1)$ & $637(29.5)$ & 0.15 \\
\hline Missing & $37(5.5)$ & $140(3.9)$ & 0.08 & $78(3.6)$ & $89(4.1)$ & 0.03 \\
\hline \multicolumn{7}{|l|}{ Oxygen saturation (\%): } \\
\hline$<93$ & $72(10.7)$ & $582(16.0)$ & 0.16 & $315(14.7)$ & $329(15.2)$ & 0.02 \\
\hline $93-96$ & $182(27.2)$ & $1147(31.6)$ & 0.10 & 669 (31.3) & 666 (30.9) & 0.01 \\
\hline$\geq 96$ & $396(59.1)$ & $1775(48.9)$ & 0.21 & $1077(50.3)$ & $1091(50.6)$ & 0.01 \\
\hline Missing & $20(3.0)$ & $123(3.4)$ & 0.02 & $80(3.7)$ & $71(3.3)$ & 0.02 \\
\hline \multicolumn{7}{|l|}{ Pulse (beats/min): } \\
\hline$<90$ & $438(65.4)$ & $2200(60.7)$ & 0.10 & $1327(62.0)$ & $1327(61.6)$ & 0.01 \\
\hline$\geq 90$ & $232(34.6)$ & $1427(39.3)$ & 0.10 & $814(38.0)$ & $828(38.4)$ & 0.01 \\
\hline \multicolumn{7}{|c|}{ Systolic blood pressure $(\mathrm{mm} \mathrm{Hg})$ : } \\
\hline$<140$ & $446(66.6)$ & $2360(65.1)$ & 0.03 & $1347(62.9)$ & $1405(65.2)$ & 0.05 \\
\hline$\geq 140$ & $224(33.4)$ & $1267(34.9)$ & 0.03 & $794(37.1)$ & $751(34.8)$ & 0.05 \\
\hline \multicolumn{7}{|l|}{ Temperature $\left({ }^{\circ} \mathrm{C}\right):$} \\
\hline$\leq 37$ & $356(53.1)$ & $1701(46.9)$ & 0.12 & $1045(48.8)$ & $1033(47.9)$ & 0.02 \\
\hline $37-37.9$ & $244(36.4)$ & $1292(35.6)$ & 0.02 & $737(34.4)$ & $771(35.8)$ & 0.03 \\
\hline 238 & $70(10.4)$ & $634(17.5)$ & 0.20 & $359(16.8)$ & $352(16.3)$ & 0.01 \\
\hline
\end{tabular}

\section{Sensitivity analyses}

Quantitative bias analysis showed that an unmeasured confounder would need to be strongly associated with receiving prophylactic anticoagulation and each outcome to explain the observed associations: lower 95\% confidence limit for e-value was 1.77 for 30 day mortality, 1.92 for inpatient mortality, and 1.46 for initiating therapeutic anticoagulation (supplementary eFigure 3). Results were robust to capping propensity scores (supplementary eTable 1), using stabilized weighting and robust variance estimation (supplementary eTable 2), extending the window for anticoagulation from 24 to 48 hours (supplementary eTable 3), and excluding direct oral anticoagulants from the exposure definition (supplementary eTable 4).

\section{Post hoc analyses}

The effect of prophylactic anticoagulation compared with no anticoagulation on 30 day mortality was similar when stratified by whether patients received subcutaneous heparin (hazard ratio 0.73, 95\% confidence interval 0.64 to 0.84$)$ or enoxaparin $(0.78$,
0.68 to 0.89 ; supplementary eTable 5). Some evidence suggested that the effect of prophylactic anticoagulation compared with no anticoagulation on 30 day mortality differed among patients who were admitted to the ICU within the first 24 hours of hospital admission (admitted to ICU: 0.91, 0.76 to 1.09; not admitted to ICU: $0.68,0.60$ to $0.77 ; \mathrm{P}=0.009$ ). Bleeding events that required transfusion were relatively rare $(n=198$, 4.6\%). Prophylactic anticoagulation compared with no anticoagulation was not associated with an increased risk of bleeding events that required transfusions $(0.87,0.71$ to 1.05$)$.

\section{Discussion}

In a nationwide cohort of 4297 patients admitted to hospital with covid-19 in the largest integrated healthcare system in the United States, initiation of predominantly heparin based prophylactic anticoagulation compared with no anticoagulation within the first 24 hours of admission was associated with a relative risk reduction of 30 day mortality as high as $34 \%$ and an absolute risk reduction of $4.4 \%$ in the context of an absolute risk of $18.7 \%$ among patients 


\begin{tabular}{|c|c|c|c|c|c|c|}
\hline \multirow[b]{2}{*}{ Laboratory findings } & \multicolumn{3}{|c|}{ Unweighted } & \multicolumn{3}{|c|}{ IPT weighted* } \\
\hline & $\begin{array}{l}\text { No anticoagulation } \\
(\mathrm{n}=670)\end{array}$ & $\begin{array}{l}\text { Prophylactic } \\
\text { anticoagulation }(n=3627)\end{array}$ & SMD & $\begin{array}{l}\text { No anticoagulation } \\
(\mathrm{n}=2141)\end{array}$ & $\begin{array}{l}\text { Prophylactic } \\
\text { anticoagulation }(n=2156)\end{array}$ & SMD \\
\hline \multicolumn{7}{|c|}{ Alanine aminotransferase $(\mathrm{U} / \mathrm{L})$ : } \\
\hline$\leq 30$ & $297(44.3)$ & $1722(47.5)$ & 0.06 & $1081(50.5)$ & $1014(47.0)$ & 0.07 \\
\hline$>30$ & $241(36.0)$ & $1456(40.1)$ & 0.09 & $751(35.1)$ & $847(39.3)$ & 0.09 \\
\hline Missing & $132(19.7)$ & $449(12.4)$ & 0.20 & $310(14.5)$ & $295(13.7)$ & 0.02 \\
\hline \multicolumn{7}{|c|}{ Aspartate aminotransferase $(\mathrm{U} / \mathrm{L})$ : } \\
\hline$\leq 30$ & $225(33.6)$ & $1171(32.3)$ & 0.03 & $717(33.5)$ & $701(32.5)$ & 0.02 \\
\hline 130 & 301 (44.9) & $1949(53.7)$ & 0.18 & $1056(49.3)$ & $1124(52.1)$ & 0.06 \\
\hline Missing & $144(21.5)$ & $507(14.0)$ & 0.20 & $367(17.2)$ & $330(15.3)$ & 0.05 \\
\hline \multicolumn{7}{|c|}{ eGFR $\left(\mathrm{mL} / \mathrm{min} / 1.73 \mathrm{~m}^{2}\right)$ : } \\
\hline$\geq 60$ & $354(52.8)$ & $2034(56.1)$ & 0.07 & $1164(54.4)$ & $1196(55.5)$ & 0.02 \\
\hline $30-59$ & $159(23.7)$ & $957(26.4)$ & 0.06 & $555(25.9)$ & $559(25.9)$ & 0.00 \\
\hline$<30$ & $82(12.2)$ & $418(11.5)$ & 0.02 & $274(12.8)$ & $252(11.7)$ & 0.03 \\
\hline Missing & 75 (11.2) & $218(6.0)$ & 0.19 & $147(6.9)$ & $149(6.9)$ & 0.00 \\
\hline \multicolumn{7}{|l|}{ Glucose (mmol/L): } \\
\hline$\leq 9.44$ & $486(72.5)$ & $2766(76.3)$ & 0.09 & $1636(76.4)$ & $1628(75.5)$ & 0.02 \\
\hline 79.44 & $135(20.1)$ & $759(20.9)$ & 0.02 & $426(19.9)$ & $449(20.8)$ & 0.02 \\
\hline Missing & $49(7.3)$ & $102(2.8)$ & 0.21 & $79(3.7)$ & $78(3.6)$ & 0.00 \\
\hline \multicolumn{7}{|l|}{ Hemoglobin $(\mathrm{g} / \mathrm{L})$ : } \\
\hline$\leq 140$ & $387(57.8)$ & $2151(59.3)$ & 0.03 & $1248(58.3)$ & $1276(59.2)$ & 0.02 \\
\hline$>140$ & $231(34.5)$ & $1353(37.3)$ & 0.06 & $812(37.9)$ & $792(36.7)$ & 0.02 \\
\hline Missing & $52(7.8)$ & $123(3.4)$ & 0.19 & $81(3.8)$ & $88(4.1)$ & 0.02 \\
\hline \multicolumn{7}{|l|}{ Platelet count $\left(\times 10^{9} / \mathrm{L}\right)$ : } \\
\hline$\leq 230$ & $421(62.8)$ & $2433(67.1)$ & 0.09 & $1472(68.8)$ & $1430(66.3)$ & 0.05 \\
\hline$>230$ & $195(29.1)$ & $1097(30.2)$ & 0.02 & $594(27.7)$ & $647(30.0)$ & 0.05 \\
\hline Missing & $54(8.1)$ & $97(2.7)$ & 0.24 & $75(3.5)$ & $78(3.6)$ & 0.01 \\
\hline \multicolumn{7}{|c|}{ White blood cell count $\left(\times 10^{9} / \mathrm{L}\right)$ : } \\
\hline$\leq 6$ & $299(44.6)$ & $1747(48.2)$ & 0.07 & $1038(48.5)$ & $1025(47.6)$ & 0.02 \\
\hline$>6$ & $319(47.6)$ & $1786(49.2)$ & 0.03 & $1031(48.2)$ & $1054(48.9)$ & 0.02 \\
\hline Missing & $52(7.8)$ & $94(2.6)$ & 0.24 & $72(3.4)$ & $76(3.5)$ & 0.01 \\
\hline \multicolumn{7}{|c|}{ Lymphocyte count $\left(\times 10^{9} / \mathrm{L}\right)$ : } \\
\hline$\leq 0.6$ & $100(14.9)$ & $600(16.5)$ & 0.04 & $353(16.5)$ & $352(16.3)$ & 0.01 \\
\hline$>0.6$ & $431(64.3)$ & $2523(69.6)$ & 0.11 & $1428(66.7)$ & $1479(68.6)$ & 0.04 \\
\hline Missing & $139(20.7)$ & $504(13.9)$ & 0.18 & $359(16.8)$ & $326(15.1)$ & 0.05 \\
\hline \multicolumn{7}{|c|}{ Total cholesterol (mmol/L): } \\
\hline$\leq 3.37$ & $152(22.7)$ & $678(18.7)$ & 0.10 & $398(18.6)$ & $414(19.2)$ & 0.02 \\
\hline 13.37 & $415(61.9)$ & $2444(67.4)$ & 0.11 & $1410(65.9)$ & $1435(66.6)$ & 0.01 \\
\hline Missing & $103(15.4)$ & $505(13.9)$ & 0.04 & $333(15.6)$ & $307(14.2)$ & 0.04 \\
\hline \multicolumn{7}{|c|}{ HDL cholesterol (mmol/L): } \\
\hline$<0.98$ & $180(26.9)$ & $917(25.3)$ & 0.04 & $551(25.7)$ & $548(25.4)$ & 0.01 \\
\hline $0.98-1.28$ & $202(30.1)$ & $1213(33.4)$ & 0.07 & $719(33.6)$ & $710(33.0)$ & 0.01 \\
\hline$\geq 1.29$ & $181(27.0)$ & $988(27.2)$ & 0.01 & $535(25.0)$ & $587(27.2)$ & 0.05 \\
\hline Missing & $107(16.0)$ & $509(14.0)$ & 0.05 & $337(15.7)$ & $311(14.4)$ & 0.04 \\
\hline \multicolumn{7}{|l|}{ Triglycerides (mmol/L): } \\
\hline$<1.55$ & $58(8.7)$ & $310(8.5)$ & 0.00 & $175(8.2)$ & $183(8.5)$ & 0.01 \\
\hline $1.55-2.32$ & $133(19.9)$ & $628(17.3)$ & 0.07 & $362(16.9)$ & $385(17.9)$ & 0.02 \\
\hline$\geq 2.33$ & $370(55.2)$ & $2165(59.7)$ & 0.09 & $1259(58.8)$ & $1268(58.8)$ & 0.00 \\
\hline Missing & $109(16.3)$ & $524(14.4)$ & 0.05 & $344(16.1)$ & $319(14.8)$ & 0.03 \\
\hline
\end{tabular}

IPT=inverse probability of treatment; SMD=absolute value of the standardized mean difference; eGFR=estimated glomerular filtration rate; $\mathrm{HDL}=$ high density lipoprotein.

*Counts after IPT weighting were calculated by multiplying weights by constant factor $k$, where $k$ was the ratio of observed sample size to number in the pseudopopulation after weighting; in this study, k=4297/8576.

who did not receive anticoagulation. These results persisted in sensitivity analyses. The evidence of benefit was strongest among patients not admitted to the ICU within the first 24 hours of admission. Additionally, severe bleeding as measured by requirement for blood transfusions was a relatively rare event and not associated with receipt of prophylactic anticoagulation. We observed similar protective effects for secondary outcomes, including inpatient mortality and initiation of therapeutic anticoagulation-a proxy for clinical deterioration that included thromboembolic events.

\section{Comparison with other evidence}

Results from previous studies investigating the role of anticoagulation among patients with covid-19 have varied. ${ }^{13} 14$ 30-37 Variations in reported associations probably derive from different definitions of anticoagulation, for both drug type and dose. Additionally, different patient populations (eg, disease specific cohorts), comparator groups, and inclusion and exclusion criteria were used. One of the largest observational studies to date reported that both prophylactic and therapeutic anticoagulation were 


\begin{tabular}{|c|c|c|c|c|c|}
\hline \multirow[b]{2}{*}{ Outcomes } & \multirow[b]{2}{*}{ No of patients } & \multirow[b]{2}{*}{ No of events } & \multirow{2}{*}{$\begin{array}{l}\text { Unweighted } \\
\text { Hazard ratio }(95 \% \mathrm{Cl})\end{array}$} & \multicolumn{2}{|c|}{ IPT weighted } \\
\hline & & & & Cumulative incidence $(95 \% \mathrm{Cl})$ & Hazard ratio $(95 \% \mathrm{Cl})$ \\
\hline \multicolumn{6}{|l|}{30 day mortality: } \\
\hline Prophylactic anticoagulation & 3627 & 513 & 0.85 (0.69 to 1.05$)$ & $14.3(13.1$ to 15.5$)$ & 0.73 (0.66 to 0.81$)$ \\
\hline No anticoagulation & 670 & 109 & Ref & 18.7 (15.1 to 22.9$)$ & Ref \\
\hline \multicolumn{6}{|l|}{ Inpatient mortality: } \\
\hline Prophylactic anticoagulation & 3627 & 418 & $0.82(0.66$ to 1.03$)$ & $11.7(10.7$ to 12.8$)$ & 0.69 (0.61 to 0.77$)$ \\
\hline No anticoagulation & 670 & 92 & Ref & 16.4 (13.0 to 20.5$)$ & Ref \\
\hline \multicolumn{6}{|c|}{ Initiating therapeutic anticoagulation: } \\
\hline Prophylactic anticoagulation & 3627 & 573 & 1.14 (0.91 to 1.42$)$ & $15.6(14.4$ to 16.8$)$ & $0.81(0.73$ to 0.90$)$ \\
\hline No anticoagulation & 670 & 92 & Ref & $18.8(15.2$ to 23.1$)$ & Ref \\
\hline
\end{tabular}

associated with a reduction in inpatient mortality by up to $55 \%$ compared with no anticoagulation across five hospitals in New York City. ${ }^{13}$ However, the study allowed patients to switch treatment groups during follow-up without comprehensively accounting for time updated confounding by indication that could have affected the results. Recently, interim results from three aggregated randomized trials encompassing 1000 inpatients with covid-19 reported study discontinuation because therapeutic doses of anticoagulation were associated with a reduction in the rate of ventilation or organ supportive interventions compared with prophylactic doses of anticoagulation. ${ }^{38}$ The interim results did not report the effect of anticoagulation on mortality, venous thromboembolism, or bleeding. Although consistent with the aggregated trial results suggesting therapeutic benefit for anticoagulation, our findings on more than 4000 inpatients extend this aggregated study suggesting benefit from prophylactic doses of anticoagulation compared with no anticoagulation, primarily in patients not requiring ICU level care. Further, we report associations with mortality and treatment intensification as well as safety data on serious bleeding events.

Our study was designed to emulate a hypothetical clinical trial, in which we used inverse probability treatment weighting to balance the distribution of covariates between treatment groups at hospital admission (analogous to randomization), defined eligibility through inclusion and exclusion criteria (eg, removed prevalent users and those with contraindications), defined time zero as the initiation of prophylactic anticoagulation, and assigned patients into treatment groups based on dose specific use of anticoagulation within 24 hours of admission. We conducted an intention-to-treat analysis because the data required to account for time updated exposures and confounders (eg, marginal structural modeling) may not be available for acute hospital admissions. For example, many hospital systems report all diagnoses that occur during a given hospital admission at discharge. Therefore, we could not determine if initiation of prophylactic anticoagulation later in hospital admission confers benefit. Multiple clinical trials are in progress to determine dosing and timing for anticoagulation during the clinical course of covid-19. ${ }^{12}$ Until clinical trial data are available, our results provide strong evidence for the use of prophylactic anticoagulation as initial treatment for patients with covid-19 on hospital admission.

Thromboembolic events in the context of covid-19 are strongly associated with mortality. ${ }^{30} 36$ 39-41 The cause of elevated thrombosis risk remains unclear, although proposed mechanisms have included systemic inflammation, endothelialitis, and activation of the complement system. ${ }^{42-44}$ Increases in a variety of inflammatory pathways, including bradykinin, interleukin $6, \mathrm{C}$ reactive protein, and growth differentiation factor 15 , have been described in covid-19. ${ }^{10} 11$ 45-54 Further, heparin has been shown to block the SARS-CoV-2 viral spike protein from binding in experimental studies. ${ }^{55-57}$ We postulate that the combination of the known antithrombotic and potential anti-inflammatory effects of heparin, 89 in addition to attenuation of viral infectivity might, at least in part, explain the observed benefit associated with prophylactic anticoagulation.

\section{Strengths and limitations of this study}

Although this study has many strengths, including the availability of detailed, longitudinal, electronic health record data on a nationwide cohort of patients admitted to hospital with covid-19, the use of rigorous pharmacoepidemiological methodology, and findings that were robust to sensitivity analyses, we recognize possible limitations. First, owing to the observational nature of the study, a degree of uncertainty persists that can only be addressed through randomized trials, which have the benefit of blinding and prospective, standardized measurement of patient characteristics, cotreatments, and outcomes. Nonetheless, we took several steps to mitigate potential confounding. We comprehensively accounted for chronic and acute health conditions at hospital admission in addition to other potential covid-19 treatments to achieve balance of these potential confounders between treatment groups. Further, we demonstrated that our results were robust to unmeasured confounding using quantitative bias analysis, which showed that an unmeasured confounder would need to be strongly associated with receipt of prophylactic anticoagulation and each of the outcomes considered to explain the observed effects. Second, information on cause of death was not available at the time of analysis. However, based 


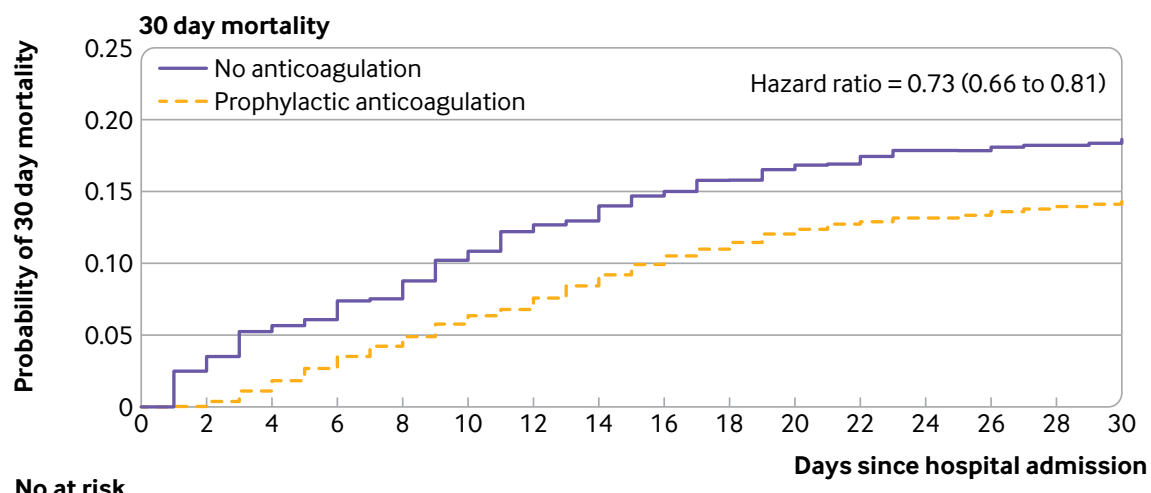

No at risk

No anticoagulation

2141208720292010198019221880186418261803178717791759175917501748

Prophylactic anticoagulation

2156215421312097206420312008197419461918189518811873186718581852

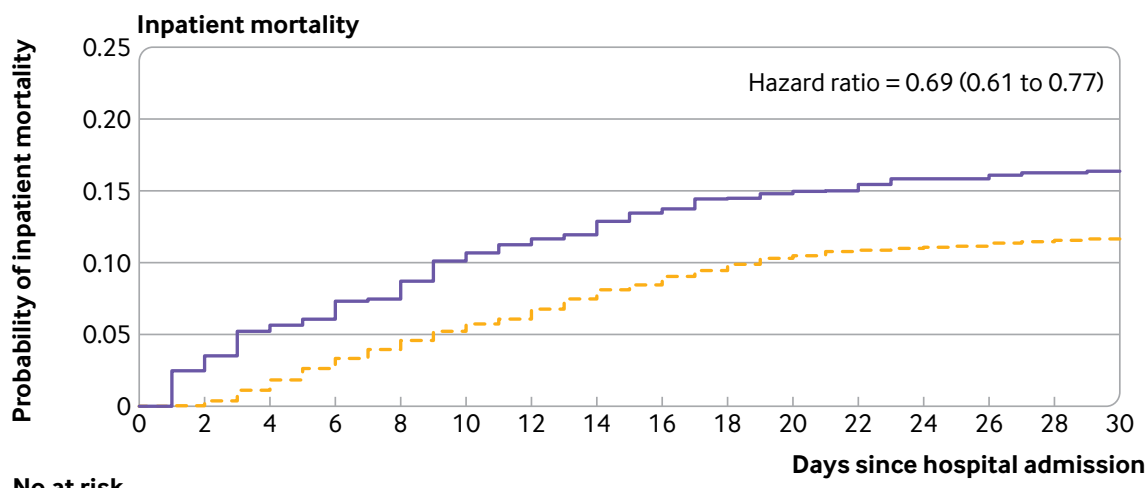

No at risk

No anticoagulation

2141208720292010198019221880186418261803178717791759175917501748

Prophylactic anticoagulation

2156215421312097206420312008197419461918189518811873186718581852

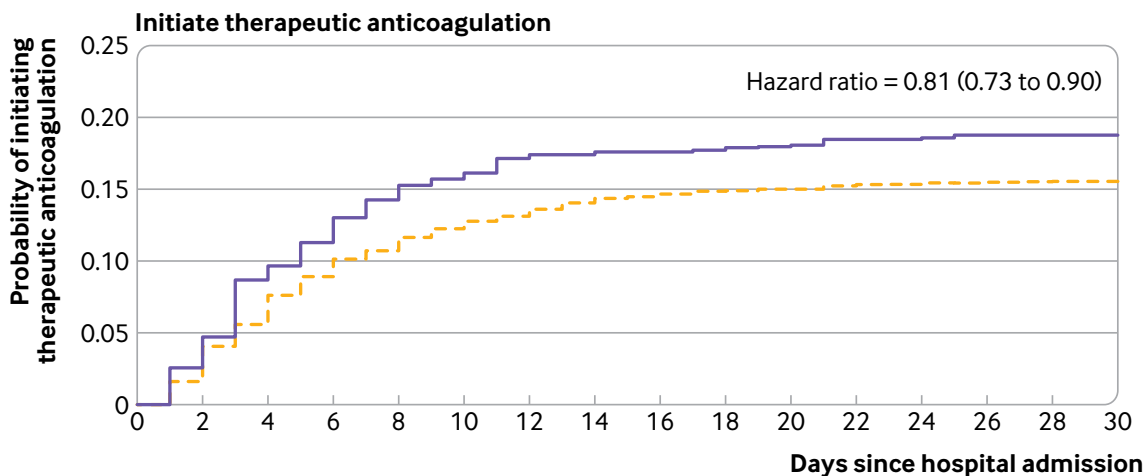

\section{No at risk}

Days since hospital admission

No anticoagulation

2141203218461778170816371587157315491533152115081496149014891489

Prophylactic anticoagulation

2156212020111914184717911759171616891664165116381632162716211617

Fig 3 | Inverse probability treatment weighted Kaplan-Meier plots. Numbers at risk were calculated by multiplying weights by constant factor $\mathrm{k}$, where $\mathrm{k}$ was the ratio of observed sample size to number in the pseudopopulation after inverse probability treatment weighting; in this study, $k=4297 / 8576$

on single center reports, ${ }^{58}$ we suspect that venous and arterial thrombosis are contributing causes of death. Third, a validated algorithm to directly identify thromboembolic events as an outcome was not available. We acknowledge that the initiation of therapeutic anticoagulation might occur as a result of many reported complications of covid-19, including venous thromboembolism, arterial thromboembolism, 
cardiac arrhythmia, and disseminated intravascular coagulation. ${ }^{59-66}$ We surmised that an intensification of anticoagulation indicated an adverse change in clinical condition, including these events. Fourth, this study was conducted on veterans currently receiving care in the Veterans Affairs healthcare system, who are older and have a higher prevalence of chronic health conditions and risk behaviors than the general US population. ${ }^{67-69}$ However, previous research has established that after adjusting for age, sex, race, ethnicity, region, and residence type, all of which were accounted for in this study, total disease burden between veterans and non-veterans does not differ. ${ }^{69}$ In separate analyses, we developed a predictive index based on Veterans Affairs data ${ }^{70}$ and have since shown that the risk of covid-19 mortality associated with age, sex, and comorbid disease diagnoses that we observed in Veterans Affairs data was consistent across other academic and national healthcare samples in the US. ${ }^{71}$ Our key finding in the current analysis has also been shown in smaller, non-veteran healthcare systems ${ }^{13} 14$; thus, effects reported in this study are probably generalizable to the wider US population. Fifth, although individuals in Veterans Affairs care represent a diversity of backgrounds, women represented a small proportion of the sample population and thus results might not be generalizable to women. Sixth, some risk factors for venous thromboembolism were not included in this dataset because they were unavailable or had high levels of missingness-for example, immobility, paresis, and biomarkers, including D dimer, $C$ reactive protein, and fibrinogen. ${ }^{72}$ Finally, we excluded patients with no history of care in the two years before study start; this only accounted for $2 \%$ of eligible patients, however, and therefore probably did not have an impact on our observed results.

\section{Conclusions and implications}

We studied a nationwide cohort of patients admitted to hospital with covid-19 and found that initiation of prophylactic, heparin based anticoagulation compared with no anticoagulation within 24 hours of admission was associated with a lower risk of 30 day mortality, in-hospital mortality, and initiation of therapeutic anticoagulation probably indicative of clinical deterioration, including thromboembolic events. This benefit seemed to be greater among patients not admitted to the ICU within 24 hours of hospital admission. Early initiation of prophylactic anticoagulation was not associated with an increased risk of bleeding that required transfusion. Our results provide strong real world evidence to support guidelines recommending the use of prophylactic anticoagulation as initial treatment for patients with covid-19 on hospital admission.

\section{AUTHOR AFFILIATIONS}

${ }^{1}$ Faculty of Epidemiology and Population Health, London School of Hygiene \& Tropical Medicine, London WC1E 7HT, UK

${ }^{2}$ VA Connecticut Healthcare System, US Department of Veterans Affairs, West Haven, CT, USA
${ }^{3}$ Cardiovascular Division, Vanderbilt University Medical Center and Vanderbilt Translational and Clinical Cardiovascular Research Center, Nashville, TN, USA

${ }^{4}$ Center for Pharmaceutical Policy and Prescribing, Health Policy Institute, University of Pittsburgh, Pittsburgh, PA, USA

${ }^{5}$ Division of General Internal Medicine, School of Medicine, University of Pittsburgh, Pittsburgh, PA, USA

${ }^{6}$ Veterans Affairs Pittsburgh Healthcare System, US Department of Veterans Affairs, Pittsburgh, PA, USA

${ }^{7}$ Center for Occupational Biostatistics and Epidemiology, Department of Biostatistics, Graduate School of Public Health, University of Pittsburgh, Pittsburgh, PA, USA

${ }^{8}$ Department of Internal Medicine, Yale School of Medicine, New Haven, CT, USA

${ }^{9}$ Department of Medicine, Vanderbilt University Medical Center and Vanderbilt Translational and Clinical Cardiovascular Research Center, Nashville, TN, USA

${ }^{10}$ Department of Neurosurgery, Yale School of Medicine, New Haven, CT, USA

${ }^{11}$ Massachusetts Veterans Epidemiology Research and Information Center (MAVERIC), VA Boston Healthcare System, Boston, MA, USA

${ }^{12}$ Faculty of Biostatistics, Department of Biostatistics, Vanderbilt University School of Medicine, Nashville, TN, USA

${ }^{13}$ Department of Medicine, Vanderbilt University Medical Center, Nashville, TN, USA

${ }^{14}$ Department of Biostatistics, Vanderbilt University Medical Center, Nashville, TN, USA

${ }^{15}$ Rocky Mountain Regional VA Medical Center, US Department of Veterans Affairs, Aurora, CO, USA

${ }^{16}$ VA Palo Alto Healthcare System, US Department of Veterans Affairs, Palo Alto, CA, USA

${ }^{17}$ Department of Medicine, Stanford University School of Medicine, Stanford, CA, USA

${ }^{18}$ Oak Ridge National Laboratory, Biosciences Division, Oak Ridge, TN, USA

${ }^{19}$ Health Services Research and Development, US Department of Veterans Affairs, Washington, DC, USA

${ }^{20}$ Center for Interdisciplinary Research on AIDS, Yale School of Public Health, New Haven, CT, USA

${ }^{21}$ Geriatric Research Education and Clinical Center, Tennessee Valley Healthcare System, US Department of Veterans Affairs, Nashville, TN, USA

The Veterans Affairs Medications, Safety, and Effectiveness Collaboratory Workgroup vetted the study design and statistical approach before study approval.

Contributors: CTR and JAB contributed equally to this work. $A C$ J and MSF are joint principal investigators. CTR, JAB, ACJ, and MSF conceived the study. CTR, CA, FK, MS, YH, MFL, and JPT curated the data. CTR performed the formal analysis. CTR, DA, ACJ, and MSF acquired funding. CTR, JAB, LT, WFG, IJD, JPT, SJWE, DA, ACJ, and MSF designed the methodology. CTR, DA, AC), and MSF managed and coordinated the project. CTR, DA, ACJ, and MSF procured resources to carry out the study. CTR, CA, FK, MS, and JPT developed programming. CTR, JAB, $\mathrm{DA}, \mathrm{AC}$, and MSF provided oversight and leadership of the project. CTR prepared data visualizations. CTR and JAB wrote the first draft of the manuscript. All the authors wrote (reviewed and edited) the manuscript. CTR and MSF are guarantors. The corresponding author attests that all listed authors meet authorship criteria and that no others meeting the criteria have been omitted.

Funding: This work was supported by the National Institute on Alcohol Abuse and Alcoholism (U01-AA026224, U24-AA020794, U01-AA020790, U10-AA013566) and by the Department of Veterans Affairs Health Services Research and Development (C19 20-405) and Office of Research and Development (MVP000). Research was also sponsored by the Laboratory Directed Research and Development Program (LOIS:10074) of Oak Ridge National Laboratory, managed by UT-Battelle, LLC for the US Department of Energy under contract DEAC05-00OR22725. The funders had no role in considering the study design or in the collection, analysis, interpretation of data, writing of the report, or decision to submit the article for publication. The views and opinions expressed in this manuscript are those of the authors and do not necessarily represent those of the Department of Veterans Affairs or the United States government. 
Competing interests: All authors have completed the ICMJE uniform disclosure form at www.icmje.org/coi disclosure.pdf and declare: This work was supported by the National Institute on Alcohol Abuse and Alcoholism (U01-AA026224, U24-AA020794, U01-AA020790, U10AA013566) and by the Department of Veterans Affairs Health Services Research and Development (C19 20-405) and Office of Research and Development (MVP000). Research was also sponsored by the Laboratory Directed Research and Development Program (LOIS:10074) of Oak Ridge National Laboratory, managed by UT-Battelle, LLC for the US Department of Energy under contract DE-AC05-000R22725. JAB reports consulting with Amgen, Bayer, JanOne, and Janssen. He serves on the data safety monitoring committee for Novartis. PMH is supported by grants from National Heart, Lung, and Blood Institute, VA Health Services Research \& Development, and University of Colorado Schoo of Medicine. He has a research agreement with Bristol-Myers Squibb through the University of Colorado. He serves as the deputy editor for Circulation: Cardiovascular Quality and Outcomes. IJD reports grants from UK National Health Service National Institute for Health Research and has received unrestricted research grants and holds shares in GlaxoSmithKline, outside of the submitted work. All other authors declare no financial relationships with any organizations that might have an interest in the submitted work in the previous three years, and no other relationships or activities that could appear to have influenced the submitted work.

Ethical approval: This study was approved by the institutional review boards of Yale University and VA Connecticut Healthcare System (ref $\#$ 0013). It has been granted a waiver of informed consent and is compliant with the Health Insurance Portability and Accountability Act.

Data sharing: Owing to US Department of Veterans Affairs (VA) regulations and our ethics agreements, the analytic data sets used for this study are not permitted to leave the VA firewall without a data use agreement. This limitation is consistent with other studies based on VA data. However, VA data are made freely available to researchers with an approved VA study protocol. For more information, please visit https://www.virec.research.va.gov or contact the VA Information Resource Center at VIReC@va.gov.

The manuscript's guarantors (CTR and MSF) affirm that the manuscript is an honest, accurate, and transparent account of the study being reported; that no important aspects of the study have been omitted; and that any discrepancies from the study as planned have been explained.

Dissemination to participants and related patient and public communities: This study was conducted under a waiver of consent, which explicitly prohibits direct patient contact. However, we have reported these findings to the Veterans Affairs central office and chief medical officers who have taken steps to implement our findings in Veterans Affairs clinical care. Study results will be shared with the public through press release, social media, and conference presentations.

Provenance and peer review: Not commissioned; externally peer reviewed.

This is an Open Access article distributed in accordance with the Creative Commons Attribution Non Commercial (CC BY-NC 4.0) license, which permits others to distribute, remix, adapt, build upon this work non-commercially, and license their derivative works on different terms, provided the original work is properly cited and the use is noncommercial. See: http://creativecommons.org/licenses/by-nc/4.0/.

1 Bikdeli B, Madhavan MV, Jimenez D, et al, Global COVID-19 Thrombosis Collaborative Group, Endorsed by the ISTH, NATF, ESVM, and the IUA, Supported by the ESC Working Group on Pulmonary Circulation and Right Ventricular Function. COVID-19 and Thrombotic or Thromboembolic Disease: Implications for Prevention, Antithrombotic Therapy, and Follow-Up: JACC State-ofthe-Art Review. J Am Coll Cardiol 2020;75:2950-73. doi:10.1016/j. jacc.2020.04.031

2 Wise J. Covid-19 and thrombosis: what do we know about the risks and treatment?BM/ 2020.369.m2058 doi:10.1136/bmi.m2058

3 Zhang R, Ni L, Di X, et al. Prevalence of venous thromboembolic events in novel coronavirus disease-2019 patients: Systematic review and meta-analysis. I Vasc Surg Venous Lymphat Disord 2020;S2213-333X(20)30654-5.

4 Kreuziger LB, Lee A, Garcia D, et al. COVID-19 and VTE/ Anticoagulation: Frequently Asked Questions (version 5.1). American Society of Hematology, https://www.hematology.org/covid-19/covid19-and-vte-anticoagulation (2020, accessed January 24, 2021).

5 Spyropoulos AC, Levy JH, Ageno W, et al, Subcommittee on Perioperative, Critical Care Thrombosis, Haemostasis of the Scientific, Standardization Committee of the International Society on Thrombosis and Haemostasis. Scientific and Standardization Committee communication: Clinical guidance on the diagnosis, prevention, and treatment of venous thromboembolism in hospitalized patients with COVID-19. I Thromb Haemost 2020;18:1859-65. doi:10.1111/jth.14929

6 Moores LK, Tritschler T, Brosnahan S, et al. Prevention, Diagnosis, and Treatment of VTE in Patients With Coronavirus Disease 2019: CHEST Guideline and Expert Panel Report. Chest 2020;158:1143-63. doi:10.1016/j.chest.2020.05.559

7 Barnes GD, Burnett A, Allen A, et al. Thromboembolism and anticoagulant therapy during the COVID-19 pandemic: interim clinical guidance from the anticoagulation forum. J Thromb Thrombolysis 2020;50:72-81. doi:10.1007/s11239-020-02138-z

8 Nasiripour S, Gholami K, Mousavi S, et al. Comparison of the Effects of Enoxaparin and Heparin on Inflammatory Biomarkers in Patients with ST-segment Elevated Myocardial Infarction: A prospective Open Label Pilot Clinical Trial. Iran J Pharm Res 2014;13:583-90.

9 Mousavi S, Moradi M, Khorshidahmad T, Motamedi M. Anti-Inflammatory Effects of Heparin and Its Derivatives: A Systematic Review. Adv Pharmacol Sci 2015;2015:507151. doi:10.1155/2015/507151

10 Li X, Li L, Shi Y, Yu S, Ma X. Different signaling pathways involved in the anti-inflammatory effects of unfractionated heparin on lipopolysaccharide-stimulated human endothelial cells. I Inflamm (Lond) 2020;17:5. doi:10.1186/s12950-020-0238-7

11 Garvin MR, Alvarez C, Miller Jl, et al. A mechanistic model and therapeutic interventions for COVID-19 involving a RAS-mediated bradykinin storm. Elife 2020;9:e59177. doi:10.7554/eLife.59177

12 Search on ClinicalTrials.gov, https://clinicaltrials.gov/ct2/ results?cond=COVID-19\&term=anticoagulation (accessed November 24, 2020).

13 Nadkarni GN, Lala A, Bagiella E, et al. Anticoagulation, Bleeding, Mortality, and Pathology in Hospitalized Patients With COVID-19.J Am Coll Cardiol 2020;76:1815-26. doi:10.1016/j.jacc.2020.08.041

14 Tang N, Bai H, Chen X, Gong J, Li D, Sun Z. Anticoagulant treatment is associated with decreased mortality in severe coronavirus disease 2019 patients with coagulopathy. J Thromb Haemost 2020;18:1094-9. doi:10.1111/jth.14817

15 Fiellin DA, Reid MC, O'Connor PG. Screening for alcohol problems in primary care: a systematic review. Arch Intern Med 2000;160:197789. doi:10.1001/archinte.160.13.1977

16 McGinnis KA, Justice AC, Tate JP, et al, VACS Project Group. Using DNA methylation to validate an electronic medical record phenotype for smoking. Addict Biol 2019;24:1056-65. doi:10.1111/adb.12670

17 Horby P, Lim WS, Emberson JR, et al, RECOVERY Collaborative Group. Dexamethasone in Hospitalized Patients with Covid-19 - Preliminary Report. N Engl J Med 2020; published online 17 July. doi:10.1056/ NEJMoa2021436.

18 Beigel JH, Tomashek KM, Dodd LE, et al, ACTT-1 Study Group Members. Remdesivir for the Treatment of Covid-19 - Final Report. N Engl I Med 2020;383:1813-26. doi:10.1056/NEJMoa2007764

19 Brookhart MA, Schneeweiss S, Rothman KJ, Glynn RJ, Avorn J, Stürmer T. Variable selection for propensity score models. Am J Epidemiol 2006;163:1149-56. doi:10.1093/aje/kwj149

20 Blake HA, Leyrat C, Mansfield KE, et al. Propensity scores using missingness pattern information: a practical guide. Stat Med 2020;39:1641-57. doi:10.1002/sim.8503

21 Blake HA, Leyrat C, Mansfield KE, Tomlinson LA, Carpenter J, Williamson EJ. Estimating treatment effects with partially observed covariates using outcome regression with missing indicators. Biom / 2020;62:428-43. doi:10.1002/bimi.201900041

22 D’Agostino RBJr, Rubin DB. Estimating and using propensity scores with partially missing data. J Am Stat Assoc 2000;95:749. doi:10.10 80/01621459.2000.10474263

23 Austin PC. Balance diagnostics for comparing the distribution of baseline covariates between treatment groups in propensity-score matched samples. Stat Med 2009;28:3083-107. doi:10.1002/ sim.3697

24 Pepe MS, Mori M. Kaplan-Meier, marginal or conditional probability curves in summarizing competing risks failure time data?Stat Med 1993;12:737-51. doi:10.1002/sim.4780120803

25 Gooley TA, Leisenring W, Crowley J, Storer BE. Estimation of failure probabilities in the presence of competing risks: new representations of old estimators. Stat Med 1999;18:695-706. 10.1002/(SICI)10970258(19990330)18:6<695::AID-SIM60>3.0.CO;2-0

26 Lau B, Cole SR, Gange SJ. Competing risk regression models for epidemiologic data. Am J Epidemiol 2009;170:244-56. doi:10.1093/aje/kwp107

27 VanderWeele TJ, Ding P. Sensitivity Analysis in Observational Research: Introducing the E-Value. Ann Intern Med 2017;167:26874. doi:10.7326/M16-2607

28 Austin PC. Variance estimation when using inverse probability of treatment weighting (IPTW) with survival analysis. Stat Med 2016;35:5642-55. doi:10.1002/sim.7084

29 Cole SR, Hernán MA. Constructing inverse probability weights for marginal structural models. Am J Epidemiol 2008;168:656-64. doi:10.1093/aje/kwn164 
30 Paolisso P, Bergamaschi L, D’Angelo EC, et al. Preliminary Experience With Low Molecular Weight Heparin Strategy in COVID-19 Patients. Front Pharmacol 2020;11:1124. doi:10.3389/fphar.2020.01124

31 Paranjpe I, Fuster V, Lala A, et al. Association of Treatment Dose Anticoagulation With In-Hospital Survival Among Hospitalized Patients With COVID-19. J Am Coll Cardiol 2020;76:122-4. doi:10.1016/j.jacc.2020.05.001

32 Salah HM, Naser JA, Calcaterra G, Bassareo PP, Mehta JL. The Effect of Anticoagulation Use on Mortality in COVID-19 Infection. Am J Cardiol 2020;134:155-7. doi:10.1016/j.amjcard.2020.08.005

33 Wijaya I, Andhika R, Huang I. The Use of Therapeutic-Dose Anticoagulation and Its Effect on Mortality in Patients With COVID-19: A Systematic Review. Clin Appl Thromb Hemost 2020;26:1076029620960797. doi:10.1177/1076029620960797

34 Rossi R, Coppi F, Talarico M, Boriani G. Protective role of chronic treatment with direct oral anticoagulants in elderly patients affected by interstitial pneumonia in COVID-19 era. Eur J Intern Med 2020;77:158-60. doi:10.1016/j.ejim.2020.06.006

35 Ayerbe L, Risco C, Ayis S. The association between treatment with heparin and survival in patients with Covid-19. J Thromb Thrombolysis 2020;50:298-301. doi:10.1007/s11239-020-02162-z

36 Ferguson J, Volk S, Vondracek T, Flanigan J, Chernaik A. Empiric Therapeutic Anticoagulation and Mortality in Critically III Patients With Respiratory Failure From SARS-CoV-2: A Retrospective Cohort Study. J Clin Pharmacol 2020;60:1411-5. doi:10.1002/jcph.1749

37 Billett HH, Reyes-Gil M, Szymanski J, et al. Anticoagulation in COVID-19: Effect of Enoxaparin, Heparin, and Apixaban on Mortality. Thromb Haemost 2020;120:1691-9. doi:10.1055/s-0040-1720978

38 Full-dose blood thinners decreased need for life support and improved outcome in hospitalized COVID-19 patients. National Heart, Lung, and Blood Institute (NHLBI), https://www.nih.gov/news-events/ news-releases/full-dose-blood-thinners-decreased-need-life-supportimproved-outcome-hospitalized-covid-19-patients (accessed January 24, 2021).

39 Zhang L, Feng X, Zhang D, et al. Deep Vein Thrombosis in Hospitalized Patients With COVID-19 in Wuhan, China: Prevalence, Risk Factors, and Outcome. Circulation 2020;142:114-28. doi:10.1161/CIRCULATIONAHA.120.046702

40 Bilaloglu S, Aphinyanaphongs Y, Jones S, Iturrate E, Hochman J, Berger JS. Thrombosis in Hospitalized Patients With COVID-19 in a New York City Health System. JAMA 2020;324:799-801. doi:10.1001/jama.2020.13372

41 Daughety MM, Morgan A, Frost E, et al. COVID-19 associated coagulopathy: Thrombosis, hemorrhage and mortality rates with an escalated-dose thromboprophylaxis strategy. Thromb Res 2020;196:483-5. doi:10.1016/j.thromres.2020.10.004

42 Carvelli J, Demaria O, Vély F, et al, Explore COVID-19 IPH group, Explore COVID-19 Marseille Immunopole group. Association of COVID-19 inflammation with activation of the C5a-C5aR1 axis. Nature 2020;588:146-50. doi:10.1038/s41586-020-2600-6

43 Ackermann M, Verleden SE, Kuehnel M, et al. Pulmonary Vascular Endothelialitis, Thrombosis, and Angiogenesis in Covid-19. N Engl J Med 2020;383:120-8. doi:10.1056/NEJMoa2015432

44 Meini S, Giani T, Tascini C. Intussusceptive angiogenesis in Covid-19: hypothesis on the significance and focus on the possible role of FGF2. Mol Biol Rep 2020;47:8301-4. doi:10.1007/s11033-02005831-7

45 Masi P, Hékimian G, Lejeune M, et al. Systemic Inflammatory Response Syndrome Is a Major Contributor to COVID-19 Associated Coagulopathy: Insights From a Prospective, Single Center Cohort Study. Circulation 2020;142:611-4. doi:10.1161/ CIRCULATIONAHA.120.048925

46 Riphagen S, Gomez X, Gonzalez-Martinez C, Wilkinson N, Theocharis P. Hyperinflammatory shock in children during COVID-19 pandemic. Lancet 2020;395:1607-8. doi:10.1016/S0140-6736(20)31094-1

47 Liu PP, Blet A, Smyth D, Li H. The Science Underlying COVID-19: Implications for the Cardiovascular System. Circulation 2020;142:6878. doi:10.1161/CIRCULATIONAHA.120.047549

48 Simpson JM, Newburger JW. Multisystem Inflammatory Syndrome in Children in Association With COVID-19. Circulation 2020;142:43740. doi:10.1161/CIRCULATIONAHA.120.048726

49 Nicolai L, Leunig A, Brambs S, et al. Immunothrombotic Dysregulation in COVID-19 Pneumonia Is Associated With Respiratory Failure and Coagulopathy. Circulation 2020;142:1176-89. doi:10.1161/ CIRCULATIONAHA.120.048488

50 Most ZM, Hendren N, Drazner MH, et al. The Striking Similarities of Multisystem Inflammatory Syndrome in Children and a Myocarditis-like Syndrome in Adults: Overlapping Manifestations of COVID-19. Circulation 2021:143:4-6. doi:10.1161/ CIRCULATIONAHA. 120.050166

51 Chau VQ, Giustino G, Mahmood K, et al. Cardiogenic Shock and Hyperinflammatory Syndrome in Young Males With COVID-19. Circ Heart Fail 2020;13:e007485. doi:10.1161/ CIRCHEARTFAILURE.120.007485
52 Myhre PL, Prebensen C, Strand H, et al. Growth Differentiation Factor 15 Provides Prognostic Information Superior to Established Cardiovascular and Inflammatory Biomarkers in Unselected Patients Hospitalized With COVID-19. Circulation 2020;142:2128-37. doi:10.1161/CIRCULATIONAHA.120.050360

53 Li X, Pan X, Li Y, et al. Cardiac injury associated with severe disease or ICU admission and death in hospitalized patients with COVID-19: a meta-analysis and systematic review. Crit Care 2020;24:468. doi:10.1186/s13054-020-03183-z

54 Allenbach Y, Saadoun D, Maalouf G, et al, DIMICOVID. Development of a multivariate prediction model of intensive care unit transfer or death: A French prospective cohort study of hospitalized COVID-19 patients. PLoS One 2020:15:e0240711. doi:10.1371/journal pone.0240711

55 Tree JA, Turnbull JE, Buttigieg KR, et al. Unfractionated heparin inhibits live wild-type SARS-CoV-2 cell infectivity at therapeutically relevant concentrations. Br J Pharmacol 2021;178:626-35. doi:10.1111/ bph.15304

56 Tandon R, Sharp JS, Zhang F, et al. Effective Inhibition of SARS-CoV-2 Entry by Heparin and Enoxaparin Derivatives. J Virol 2021;95:e01987-20.

57 Clausen TM, Sandoval DR, Spliid CB, et al. SARS-CoV-2 Infection Depends on Cellular Heparan Sulfate and ACE2. Cell 2020:183:1043-1057.e15. doi:10.1016/i.cell.2020.09.033

58 Poissy J, Goutay J, Caplan M, et al, Lille ICU Haemostasis COVID-19 Group. Pulmonary Embolism in Patients With COVID-19: Awareness of an Increased Prevalence. Circulation 2020;142:184-6. doi:10.1161/CIRCULATIONAHA.120.047430

59 Singh B, Kaur P, Ajdir N, Gupta S, Maroules M. Covid-19 Presenting as Acute Limb Ischemia. Cureus 2020;12:e9344.

60 Naudin I, Long A, Michel C, et al. Acute aortoiliac occlusion in a patient with novel coronavirus disease-2019. J Vasc Surg 2021;73:18-21. doi:10.1016/j.jvs.2020.10.018

61 Bozzani A, Arici V, Tavazzi G, et al. Acute arterial and deep venous thromboembolism in COVID-19 patients: Risk factors and personalized therapy. Surgery 2020;168:987-92. doi:10.1016/j. surg.2020.09.009

62 Al-Samkari H, Karp Leaf RS, Dzik WH, et al. COVID-19 and coagulation: bleeding and thrombotic manifestations of SARS-CoV-2 infection. Blood 2020;136:489-500. doi:10.1182/blood.2020006520

63 Helms I, Tacquard C, Severac F, et al, CRICS TRIGGERSEP Group (Clinical Research in Intensive Care and Sepsis Trial Group for Global Evaluation and Research in Sepsis). High risk of thrombosis in patients with severe SARS-CoV-2 infection: a multicenter prospective cohort study. Intensive Care Med 2020;46:1089-98. doi:10.1007/ s00134-020-06062-x

64 Peltzer B, Manocha KK, Ying X, et al. Outcomes and mortality associated with atrial arrhythmias among patients hospitalized with COVID-19. J Cardiovasc Electrophysiol 2020;31:3077-85. doi:10.1111/jce.14770

65 Lala A, Johnson KW, Januzzi JL, et al, Mount Sinai COVID Informatics Center. Prevalence and Impact of Myocardial Injury in Patients Hospitalized With COVID-19 Infection. J Am Coll Cardiol 2020:76:533-46. doi:10.1016/i.jacc.2020.06.007

66 Gawałko M, Kapłon-Cieślicka A, Hohl M, Dobrev D, Linz D. COVID-19 associated atrial fibrillation: Incidence, putative mechanisms and potential clinical implications. Int / Cardiol Heart Vasc 2020;30:100631. doi:10.1016/j.ijcha.2020.100631

67 Hoerster KD, Lehavot K, Simpson T, McFall M, Reiber G, Nelson KM. Health and health behavior differences: U.S. Military, veteran, and civilian men. Am J Prev Med 2012;43:483-9. doi:10.1016/j. amepre.2012.07.029

68 Lehavot K, Hoerster KD, Nelson KM, Jakupcak M, Simpson TL. Health indicators for military, veteran, and civilian women. Am I Prev Med 2012;42:473-80. doi:10.1016/j.amepre.2012.01.006

69 RAND Health. Current and projected characteristics and unique health care needs of the patient population served by the Department of Veterans Affairs. RAND Corporation, 2015.

70 King JTJr, Yoon JS, Rentsch CT, et al. Development and validation of a 30-day mortality index based on pre-existing medical administrative data from 13,323 COVID-19 patients: The Veterans Health Administration COVID-19 (VACO) Index. PLoS One 2020;15:e0241825. doi:10.1371/journal.pone.0241825

71 King JTJr, Yoon JS, Bredl ZM, et al. Accuracy of the Veterans Health Administration COVID-19 (VACO) Index for predicting short-term mortality among 1,307 Yale New Haven Hospital inpatients and 427,224 Medicare patients.medRxiv. Epub ahead of print January 4, 2021. doi:10.1101/2021.01.01.20249069

72 Darzi AJ, Karam SG, Charide R, et al. Prognostic factors for VTE and bleeding in hospitalized medical patients: a systematic review and meta-analysis. Blood 2020;135:1788-810. doi:10.1182/ blood.2019003603

Supplementary information: additional boxes, figures, and tables, and STROBE and RECORD statements 